\title{
Attenuation of polyglutamine-induced toxicity by enhancement of mitochondrial OXPHOS in yeast and fly models of aging
}

\author{
Andrea L. Ruetenik ${ }^{1,2,3}$, Alejandro Ocampo ${ }^{1,2,3, \pi}$, Kai Ruan ${ }^{4,5, \#}$, Yi Zhu ${ }^{4,5}$, Chong Li ${ }^{4,6}$, R. Grace Zhai ${ }^{1,4,5,6}$ and \\ Antoni Barrientos ${ }^{1,2,3,5, *}$ \\ ${ }^{1}$ Neuroscience Graduate Program, University of Miami Miller School of Medicine, Miami, FL 33136, USA. \\ ${ }^{2}$ Department of Neurology, University of Miami Miller School of Medicine, Miami, FL 33136, USA. \\ ${ }^{3}$ Department of Biochemistry and Molecular Biology, University of Miami Miller School of Medicine, Miami, FL 33136, USA. \\ ${ }^{4}$ Department of Molecular and Cellular Pharmacology, University of Miami Miller School of Medicine, Miami, FL 33136, USA. \\ ${ }^{5}$ Molecular and Cellular Pharmacology Graduate Program, University of Miami Miller School of Medicine, Miami, FL 33136, USA. \\ ${ }^{6}$ Human Genetics and Genomics Graduate Program, University of Miami Miller School of Medicine, Miami, FL 33136, USA. \\ "Current Address: Gene Expression Laboratory. The Salk Institute for Biological Studies, La Jolla, California 92037, USA. \\ \# Current Address: Department of Neurology, School of Medicine, Johns Hopkins University, Maryland 21205, USA. \\ * Corresponding Author: \\ Antoni Barrientos, Departments of Neurology and Biochemistry \& Molecular Biology, University of Miami Miller School of Medicine; \\ RMSB 2067, 1600 NW 10th Ave., Miami, FL 33136, USA; Tel: +1 305243 8683; Fax: +1 305243 7404; E-mail: \\ abarrientos@med.miami.edu
}

\begin{abstract}
Defects in mitochondrial biogenesis and function are common in many neurodegenerative disorders, including Huntington's disease (HD). We have previously shown that in yeast models of HD, enhancement of mitochondrial biogenesis through overexpression of Hap4, the catalytic subunit of the transcriptional complex that regulates mitochondrial gene expression, alleviates the growth arrest induced by expanded polyglutamine (polyQ) tract peptides in rapidly dividing cells. However, the mechanism through which HAP4 overexpression exerts this protection remains unclear. Furthermore, it remains unexplored whether HAP4 overexpression and increased respiratory function during growth can also protect against polyQ-induced toxicity during yeast chronological lifespan. Here, we show that in yeast, mitochondrial respiration and oxidative phosphorylation (OXPHOS) are essential for protection against the polyQ-induced growth defect by HAP4 overexpression. In addition, we show that not only increased HAP4 levels, but also alternative interventions, including calorie restriction, that result in enhanced mitochondrial biogenesis confer protection against polyQ toxicity during stationary phase. The data obtained in yeast models guided experiments in a fly model of HD, where we show that enhancement of mitochondrial biogenesis can also protect against neurodegeneration and behavioral deficits. Our results suggest that therapeutic interventions aiming at the enhancement of mitochondrial respiration and OXPHOS could reduce polyQ toxicity and delay disease onset.
\end{abstract}

doi: 10.15698/mic2016.08.518 Received originally: 17.05.2016; in revised form: 23.06.2016, Accepted 04.07.2016, Published 26.07.2016.

Keywords: Saccharomyces cerevisiae, mitochondrial respiration, mitochondrial OXPHOS, mitochondrial biogenesis, polyglutamine toxicity, yeast chronological life span, Drosophila model, caloric restriction.

\section{Abbreviations:}

CLS - chronological life span, polyQ - polyglutamine,

$O E$-overexpression,

OXPHOS - oxidative phosphorylation.

\section{INTRODUCTION}

Metabolic and mitochondrial abnormalities are a prominent feature of aging and neurodegeneration, which is not surprising, given the high-energy demands of neuronal function. Mitochondria perform multiple roles essential for cellular metabolism and physiology. Their main function is the conversion of energy stored in nutrients into chemical energy, aerobically, through oxidative phosphorylation (OXPHOS), which couples oxygen reduction by the mitochondrial respiratory chain (MRC) to ATP synthesis.

Neurodegenerative diseases are frequently caused by the gain-of-toxic-function of disease-specific proteins that 
misfold and oligomerize, affecting the integrity and function of selective neuronal systems. Despite toxic protein heterogeneity, mounting evidence suggest that mitochondrial dysfunction and oxidative stress occur early in all major neurodegenerative diseases [1], including $\alpha$ synucleopathies, taupathies, and polyglutamine (polyQ) disorders. PolyQ disorders, including HD and spinocerebellar ataxias, are caused by a CAG codon repeat expansion in disease-specific genes resulting in the expression of misfolding/aggregation-prone proteins with expanded polyQ stretches. Mitochondrial dysfunction, altered mitochondrial dynamics and impaired axonal trafficking have been associated with the pathogenesis of polyQ diseases in human patients and several research model organisms [2, 3]. In addition, mutant huntingtin $(\mathrm{Htt})$ may damage neurons directly by inducing mitochondrial depolarization and altering calcium homeostasis in patients and in mouse models [2]. Finally, mutant $\mathrm{Htt}$ has been shown to alter mitochondrial function indirectly by inhibiting expression of the transcriptional co-activator PGC- $1 \alpha$ (peroxisome proliferator-activated receptor (PPAR)-gamma-coactivator $1 \alpha$, which regulates several metabolic processes including mitochondrial biogenesis and respiration when interacting with the respiratory factors NRF1 and NRF2 [3].

Due to the complexity of proteotoxic neuronal death, development of suitable models is critical to dissect the precise mechanism/s in the disease process and the aging/disease relationship. Whereas several mammalian cell culture models have been developed over the past 15 years, the unicellular yeast model Saccharomyces cerevisiae and its powerful genetics has also been successfully used to study the protein aggregation associated with polyQ disorders [4-7]. Although yeast cells lack many of the structural and functional hallmarks of neuronal cells that are critical for the progression of neurodegeneration, yeast models have provided a platform to elucidate the basic cellular mechanisms of toxicity triggered by human neurotoxic proteins and to identify targets for therapeutic intervention [7-9]. In S. cerevisiae, expression of $\mathrm{Htt}$ exon-1 fragments comprising the polyQ stretches faithfully recapitulates $\mathrm{Htt}$ misfolding/aggregation in a polyQ lengthdependent manner [10]. Upon polyQ toxicity, several pathways, including ER stress, cytoskeletal disturbances, oxidative stress, and mitochondrial dysfunction contribute to growth arrest and cell death [4, 6, 11]. Genetic or pharmacological interventions aiming to protect each of these pathways from disruption have been shown to confer protection against polyQ toxicity. Specifically, we have shown that in growing yeast cultures, polyQ-induced toxicity can be suppressed by enhancement of mitochondrial biogenesis, achieved by overexpression of Hap4, the catalytic subunit of the transcriptional complex Hap2,3,4,5 that globally activates transcription of nuclear genes involved in mitochondrial respiration [12]. The specific mechanism involved in the suppression remains to be fully elucidated.

As a handicap for the currently available yeast models of proteopathies, all studies reported to date have been conducted on rapidly dividing mitotic cells, and have manifested acute toxicity based on the high galactose-inducible expression of toxic proteins. Only a single study has looked at yeast survival in the stationary phase of yeast expressing $\alpha$-synuclein, also from a galactose-inducible promoter [13]. Nevertheless, in this report, $\alpha$-synuclein expression was induced at the moment of inoculation, severely restricting survival during the exponential phase and diauxic shift. While studies in dividing yeast are interesting and have provided useful information into the diverse cellular processes that mutant proteins can alter, these models fail to mirror the post-mitotic state of neurons in the adult brain. Furthermore, due to the fact that neurodegenerative disorders are age-associated disorders, we believe that disease models that facilitate the study of proteotoxicity in the context of aging will be more suitable for the better understanding of these kinds of diseases. These considerations support yeast chronological aging as a good model to study mitochondrial function alterations involved in aging and age-related disorders [8].

The yeast chronological life span (CLS) model of aging measures the capacity of stationary $\left(\mathrm{G}_{0}\right)$ cultures to maintain viability over time (1 to several weeks) [14]. The CLS model of aging is an established model for the regulation of aging in post-mitotic mammalian cells, such as neurons [8]. For CLS studies, yeast cells are usually aged in media containing $2 \%$ glucose. Under these conditions, cells divide exponentially producing energy preferentially by fermentation while respiration is repressed in a glucose concentration-dependent manner. As glucose is being consumed, growth slows down and the diauxic shift occurs, which involves a shift from fermentation to respiration. In addition, the activation of stress resistance mechanisms and the accumulation of nutrient stores (glycogen and trehalose) allow the survival in the stationary phase where the metabolic rate is significantly reduced. Mitochondrial respiration during growth is known to be essential for a strain to achieve a standard wild-type CLS [15]. However, yeast cells have a large reserve respiratory capacity to sustain CLS, as respiration only limits CLS when depleted below a threshold of $\sim 40 \%$ of wild-type respiration [16]. Strains that respire below this threshold during growth have extremely poor respiratory capacity in the stationary phase and rapidly consume their nutrient stores due to the inefficient production of energy through fermentation, resulting in a very short CLS [16]. How polyQ expression interferes with yeast CLS remains unclear.

To study polyQ toxicity in the context of aging and its suppression by enhancement of mitochondrial biogenesis, we have created flexible beta-estradiol inducible yeast models that allow for the tight regulation of toxic protein expression at any moment of growth. Our data show that boosted mitochondrial respiration during growth is essential for Hap4-induced suppression of polyQ toxicity during CLS and that several genetic and nutritional interventions that enhance mitochondrial respiration during growth and extend life span effectively alleviate polyQ toxicity during $\mathrm{CLS}$. These data has been subsequently validated in a Drosophila model of $\mathrm{HD}$, where overexpression of a PGC1- $\alpha$ homolog also protects against neurodegeneration and behavioral deficits. Our results further suggest that therapeu- 
tic interventions that enhance mitochondrial OXPHOS could reduce polyQ toxicity and delay disease onset in patients.

\section{RESULTS}

Development of yeast models of polyQ cytotoxicity in post-mitotic cells.

In order to study proteotoxicity in post-mitotic yeast using the CLS assay, we generated metabolism-independent inducible models of polyQ toxicity. To this end, we constucted and tested several candidate expression systems [17], and ultimately chose an optimized $\beta$ estradiol-inducible system modified from [18] as the system that performed best in our conditions (Figure 1A). This expression system is based on the constitutive expression of a transactivator fusion protein GAL4.ER.VP16, which is formed by a GAL4 DNA binding domain, a $\beta$-estradiol receptor domain and a VP16 (virus protein 16) transcriptional activator that can activate transcription of a gene placed under the control of a galactose-inducible promoter
A

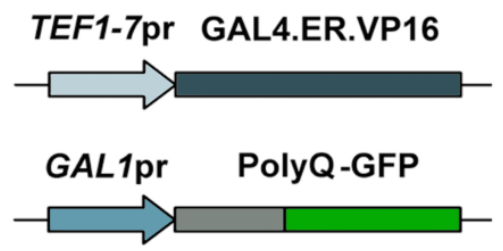

B

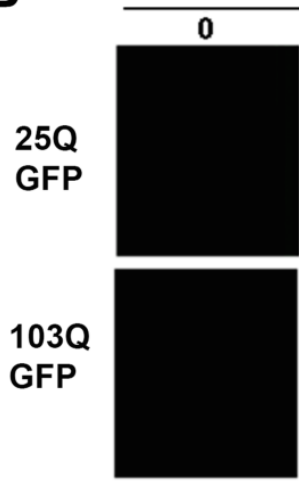

Days in stationary phase
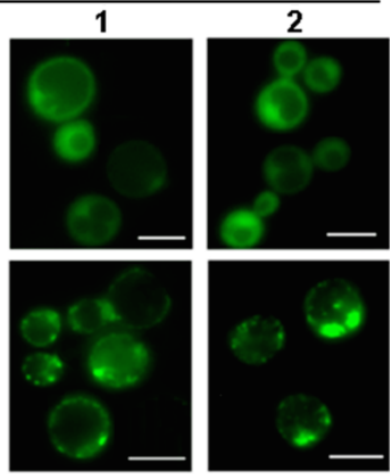

$50 \mathrm{nM} \beta$-estradiol added at day 0
C

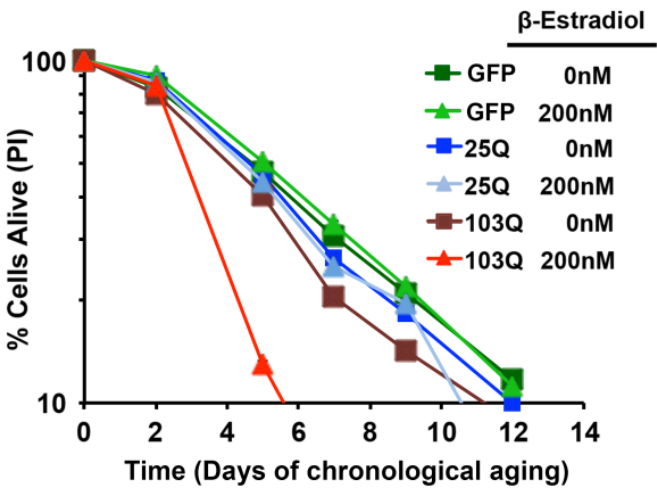

D

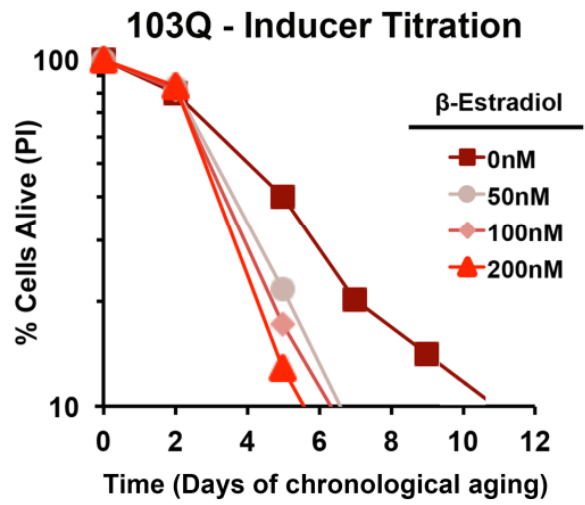

E

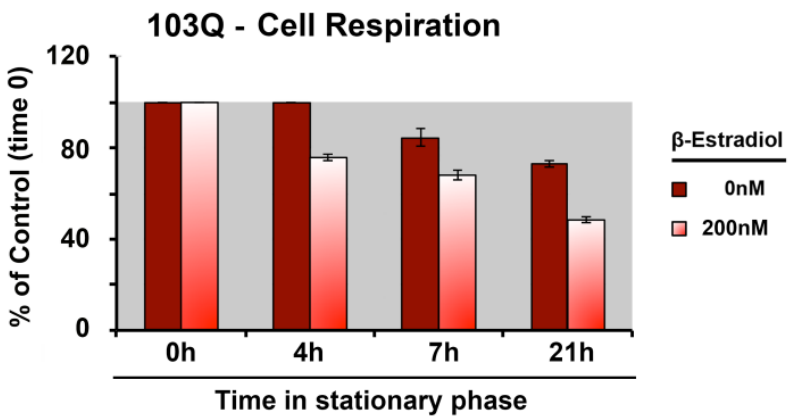

FIGURE 1: Generation of inducible yeast models of proteopathies. (A) Schematic of polyQ-GFP expression cassettes under the control of the $\beta$-estradiol inducible promoter. (B) Chronology of polyQ-GFP protein accumulation, followed by fluorescence microscopy, in cells induced with $50 \mathrm{nM} \beta$-estradiol. The bar is $5 \mu \mathrm{m}$. (C) and (D) Yeast CLS. Survival of wild-type cells expressing 25Q or 103Q from a $\beta$-estradiolinducible promoter activated with the indicated amounts of inducer or supplemented with the solvent (ethanol) was estimated by propidium iodide (PI) staining and flow cytometry analysis of 10,000 cells. Data is an average of three samples in \% of cells alive at day 0 ( $72 \mathrm{~h}$ after inoculation). In (D) a $\beta$-estradiol titration was performed. (E) Time-course of endogenous cell respiration during the first day in stationary phase of growth of non-induced and induced $103 Q$ cultures. 
(GAL1pr). In the absence of the hormone, the fusion protein is repressed by the yeast chaperones from the Hsp90 family [18]. Upon media supplementation with $\beta$-estradiol, the fusion protein is de-repressed, binds to GAL1pr through the GAL4 DNA binding domain and the VP16 recruits the transcriptional machinery to start transcription of the gene of interest.

Using this system, we created models expressing 25Q, $46 \mathrm{Q}, 72 \mathrm{Q}$ or $103 \mathrm{Q} \mathrm{N}$-terminal Huntingtin ( $\mathrm{Htt}$ ) exon-1 fragment proteins, and have confirmed that polyQ expression is well-induced with a low $\beta$-estradiol concentration (50 nM) upon transition to the stationary phase (see $25 Q$ and 1030 in Figure 1B) without detectable leakage of the system before induction (Figure 1B and [17]). For the chronological life span (CLS) experiments described herein, wild-type cells expressing 25Q- and cells expressing highly-pathogenic 103Q-N-terminal $\mathrm{Htt}$, under the control of the described $\beta$ estradiol inducible promoter, were pre-grown in minimum media containing $2 \%$ glucose until they reached stationary phase (72 hours). At this moment, protein expression was induced by addition of $\beta$-estradiol. After induction, polyQ protein accumulates in a diffused distribution in the cytoplasm of 25Q-expressing cells, while $103 Q$ fragments form insoluble protein aggregates over time (Figure 1B). When expression was induced with $200 \mathrm{nM} \beta$-estradiol, expression of GFP or 250 did not produce any measurable effect in CLS, as determined by flow cytometry after PI staining, whereas 1030 produced a toxic effect that curtails maximum yeast CLS by $50 \%$, (Figure $1 \mathrm{C}$ ). These results were in agreement with the analysis of cellular viability using a clonogenic assay based on counting of CFU (colony for- mation units, Figure S1). A titration of the inducer indicated that polyQ expression is well induced and only slightly increases further in a dose-dependent manner with concentrations from $50 \mathrm{nM}$ to $200 \mathrm{nM}$, although $103 \mathrm{Q}$ toxic effect on CLS was similar in this concentration range (Figure 1D). As a control, no toxic effects of the hormone were observed (not shown).

PolyQ cytotoxicity in chronologically aging yeast is suppressed by genetically-driven enhancement of mitochondrial biogenesis

We have previously reported that cytotoxicity of a mutant huntingtin fragment in yeast involves early alterations in mitochondrial OXPHOS function, loss of membrane potential and ROS generation [4, 5]. Here, we have observed that 1030 expression induced at day 0 of chronological life span resulted in $25 \%$ lowered endogeneous cell respiration after $4 \mathrm{~h}$ in stationary phase, when all the cells are still alive (Figure 1E). Although respiration of non-induced cells also decreased over time, induced cells were decreased by $35 \%$ after $20 \mathrm{~h}$ in stationary phase (Figure $\mathbf{1 E}$ and $\mathbf{2 A}$ ). As a toxicity suppressor mechanism, we have shown earlier that enhancement of mitochondrial biogenesis through overexpression (OE) of Hap4, the catalytic subunit of the HAP transcriptional complex that serves as the master regulator of nuclear genes encoded mitochondrial proteins in yeast [12], confers robust protection against the 103Qinduced growth deficit during the exponential phase [4]. Therefore, we hypothesized that HAP4 may also confer protection against $103 \mathrm{Q}$-induced toxicity in the stationary
A

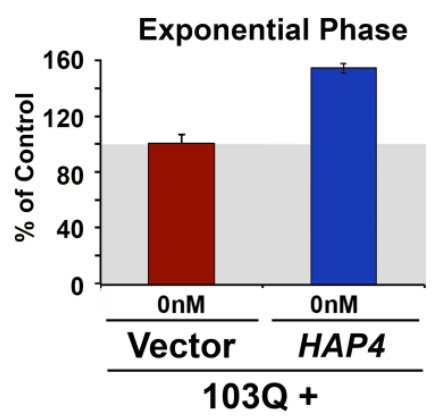

Endogenous Cell Respiration

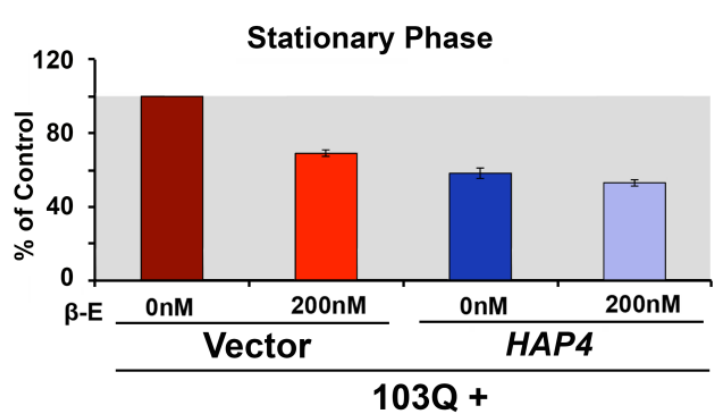

B

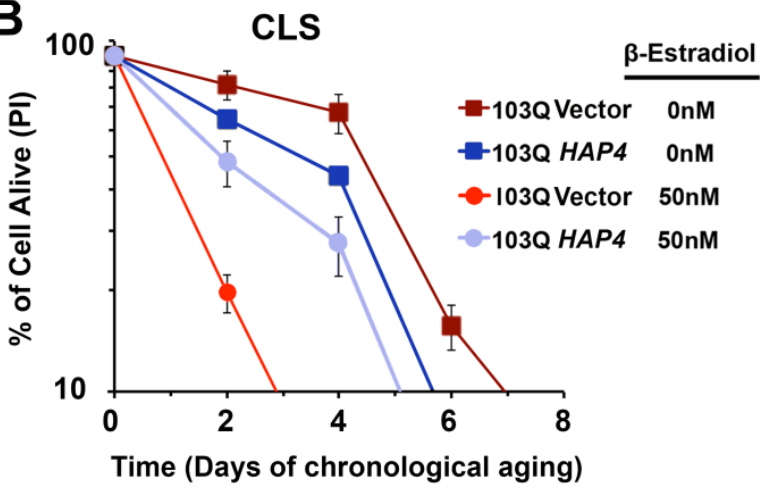

FIGURE 2: PolyQ-induced toxicity in CLS models of yeast aging is suppressed by HAP4 overexpression-mediated increase in mitochondrial biogenesis. (A) Endogenous cell respiration during the exponential and stationary phases of growth of noninduced and induced 103Q cultures overexpressing or not HAP4. Bars represent mean \pm S.D., $n=3$. (B) Effect of increased mitochondrial biogenesis by HAP4 overexpression on CLS of yeast expressing 1030 from day 0 in the stationary phase. Error bars represent S.E.M. for 3 independent experiments. (C) PolyQ expression levels at day 1 after $\beta$-estradiol induction estimated by flow cytometry quantification of GFP fluorescence. 
phase.

To test this hypothesis, 1030 cells and $103 Q$ cells carrying a construct that overexpresses HAP4 under the control of its own promoter were grown in media containing $2 \%$ glucose and 1030 expression was induced upon transition to the stationary phase. As previously reported [16], non-induced wild-type-like cells constitutively overexpressing HAP4 respired at a rate of $150 \%$ of wild-type during growth and $50 \%$ of wild-type during the stationary phase (Figure 2A). For clarification, wild-type strains have a reduced metabolic rate when they reach the stationary phase, and their respiratory rate at day 0 of CLS (72 hr after inoculation) - here considered as $100 \%$ - is actually $20 \%$ of their exponential-phase respiratory rate [16]

HAP4 OE was previosuly reported to slightly extend CLS when the cells were transferred to water upon reaching the stationary phase [19]. However, under our experimental conditions, enhanced mitochondrial biogenesis and respiration during growth was not sufficient to extend maximum CLS in non-induced cells [16] (Figure 2B). On the contrary, enhanced mitochondrial biogenesis was able to prevent the decline in respiratory capacity of 103Q-expressing cells after 20 hours in exponential phase (Figure 2A) and, most importantly, provide a CLS extension to yeast expressing 103Q, from 3 to 5 days (Figure 2B). This suppression of 1030 toxicity was not due to decreased 1030 protein, as polyQ-GFP fluorescence was indistinguishable between cells with or without HAP4 expression (Figure 2C). We envision that by increasing mitochondrial biogenesis during growth, the cell becomes equipped with a larger buffering system against 103Qinduced mitochondrial damage later during CLS [4]. Furthermore, preserving aerobic energy conversion is expected to have a positive impact in ATP-dependent 1030 clearance pathways and refolding chaperone systems.

PolyQ cytotoxicity in chronologically aging yeast is attenuated by nutritional respiratory preconditioning

Yeast CLS has been reported to be extended by increasing the levels of cellular respiration during the initial exponential growth phase achieved by culture on a respiratory carbon source $[4,16,19-22]$ or by pre-culturing the cells in media containing $0.5 \%$ rather than $2 \%$ glucose (caloric restriction or CR) $[16,23-25]$. Although the mechanisms involved in the two models are very different, as explained below, we wanted to test whether suppression of 1030 toxicity during CLS could also be confered by these interventions known to enhance mitochondrial respiration and biogenesis.

First, we started by modifying the synthetic medium from containing glucose (WOGLU), a fermentable carbon source, to containing the non-fermentable carbon sources ethanol and glycerol (WOEG). In the presence of glucose, mitochondrial biogenesis is repressed in a concentrationdependedt manner through inhibition of the HAP complex [26]. On the contrary, growth in WOEG media results in HAP-mediated increase in mitochondrial biogenesis, including components of the mitochondrial respiratory chain and oxidative phosphorylation system [27, 28],
A

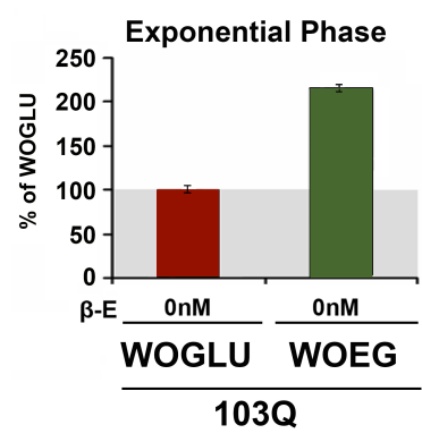

Endogenous Cell Respiration
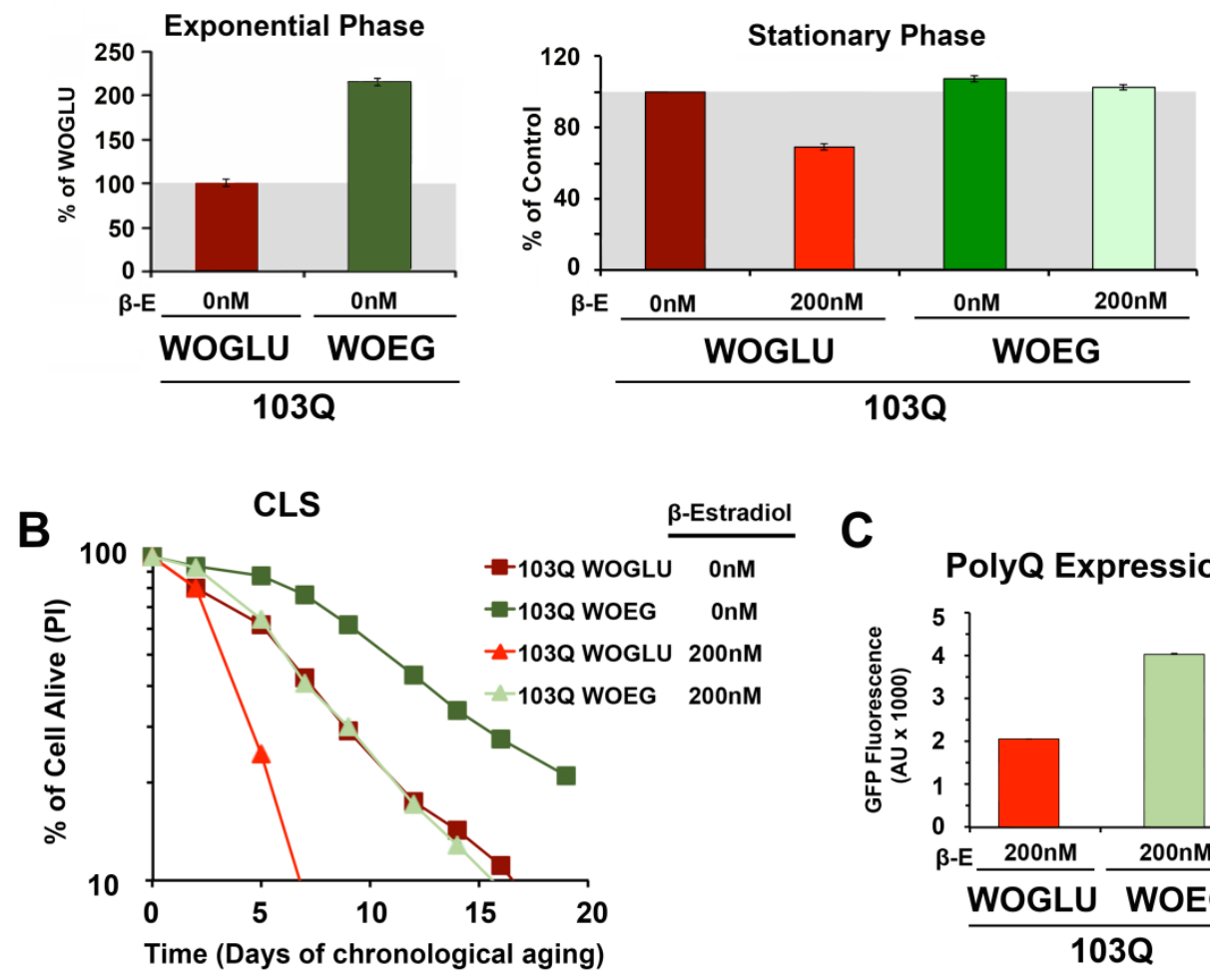

FIGURE 3: PolyQ-induced toxicity in CLS models of yeast aging is minimized by preconditioning the cells in respiratory media. (A) Endogenous cell respiration during the exponential and stationary phases of growth of noninduced and induced 103Q cultures grown in minimum synthetic media containing either the fermentable glucose (WOGLU) or the nonfermentable ethanol-glycerol (WOEG) as the carbon sources. Error bars are S.D., $\mathrm{n}=3 .{ }^{*} P<$ $0.05, * * p<0.005$. (B) Effect of growth in WOEG respiratory media on $103 Q$ yeast CLS compared to WOGLU media. S.D $<1$ for all samples, $\mathrm{n}=3$. (C) PolyQ expression levels at day 1 of CLS after $\beta$-estradiol induction estimated by flow cytometry quantification of GFP fluorescence. 
similar to what is achieved by overexpression of HAP4. This was reflected by a 2-fold higher endogenous cell respiration during growth in WOEG than in WOGLU (Figure 3A). Similarily to the results obtained with HAP4 OE, growth in WOEG media suppressed $103 \mathrm{Q}$ toxicity by preventing the decline in respiratory capacity of 1030 expressing cells after $20 \mathrm{~h}$ in exponential phase (Figure 3A) leading to a 2.3-fold increase in maximum survival of 1030expressing cells (Figure 3B). This protective effect was not due to a reduction in the levels of polyQ protein that were actually higher in WOEG media compared to WOGLU, as estimated by GFP fluroescence (Figure 3C). The CLS extension conferred by growth in respiratory carbon sources has been suggested to be a reflection of efficient survival in stationary phase requiring respiratory metabolism [29] and a reduction in medium acidification [21]. Furthermore, respiration-adapted cultures, do not just display maximal longevity, but also maintain full replicative lifespan during CLS [19]. Regarding 103Qexpressing cells, we envision, as for HAP4 OE cultures, that enhancement of mitochondrial biogenesis and respiration, could minimize the effect of 103Q-induced mitochondrial damage and might enhance the activity of $103 \mathrm{Q}$ refolding chaperone systems.

Subsequently, we decided to test whether caloric restriction (CR) could also suppress 1030 toxicity. In yeast, $\mathrm{CR}$, modeled by growing cells in media with $0.5 \%$ glucose, has been shown to result in a Hap4-dependent enhancement of mitochondrial biogenesis, as well as an increase in respiration (Figure 4A and [16]) and ROSadaptive signaling during growth [16]. CR extends CLS in part through the downregulation of signaling pathways involving nutrient-responsive kinases, such as Ras/CAMP/PKA, TOR, and Sch9, which promote the expression of stress-response genes and the accumulation of storage carbohydrates $[16,23,25]$. We recently reported that CR-induced extension of CLS required the cells to have a minimum threshold respiratory capacity (40\% of wild-type) during exponential growth in nonrestricted WOGLU media [16]. In wild-type cells [16] or in our non-induced 1030 cells (Figure 4A), CR led to a $30 \%$ higher respiratory rate during exponential growth but, importantly, completely abolished the need for respiration in the stationary phase. CR involves a metabolic remodeling that includes the shift from fermentation to respiration, which is essential for CLS extension [24]. However, the metabolic remodeling that occurs during the diauxic shift allows CR cells to dramatically reduce their metabolic rate in the stationary phase and hence consume their stored nutrients at a rate slower than that of cells grown in $2 \%$ glucose $[16,30]$. Furthermore, in stationary phase, CR cells have lowered oxidative stress [16, 20], which may contribute to CLS extension.

As expected, CR-treated non-induced cells had a markedly longer lifespan than those grown without the intervention (Figure 4B), which is in agreement with previous reports showing that, in wild-type cells, CR led to a more than 2-fold extension of their maximum CLS to 27 days [16, 23]. Induction of 103Q-expression at day 0 of CLS in CR-treated cells was still toxic and reduced maximum CLS to slightly over 20 days. However, attention should be focused on the fact that 103Q-expressing cells grown in CR media increased cell survival from 7 days to more than 20 days, an extenstion over that of even non-induced cells
A

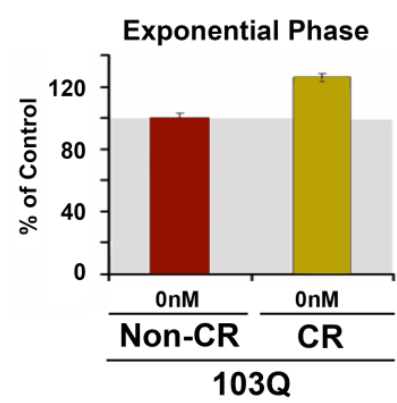

Endogenous Cell Respiration
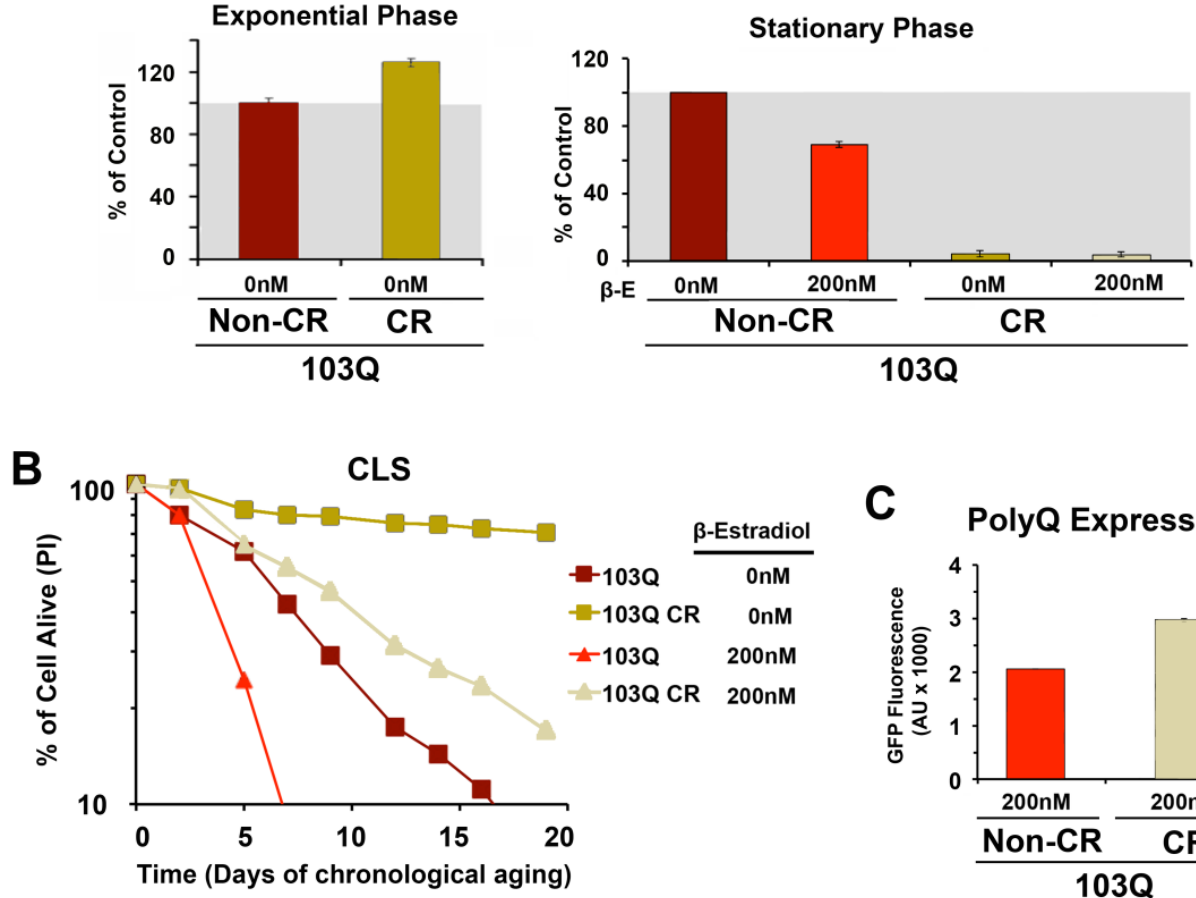

FIGURE 4: PolyQ-induced toxicity in CLS models of yeast aging is markedly suppressed by preconditioning the cells in $\mathbf{0 . 5 \%}$ glucose-containing media (caloric restriction or CR). (A) Endogenous cell respiration during the exponential and stationary phases of growth of noninduced and induced 1030 cultures grown in minimum synthetic (WOGLU) media containing either $2 \%$ (control) or $0.5 \%$ (CR) glucose as the carbon source. Bars represent mean \pm S.D., $\mathrm{n}=3$. (B) Effect of CR in 1030 yeast CLS. S.D $<1$ for all samples, $\mathrm{n}=3$. (C) Poly $\mathrm{Q}$ expression levels at day 1 after $\beta$ estradiol induction estimated by flow cytometry quantification of GFP fluorescence.
C

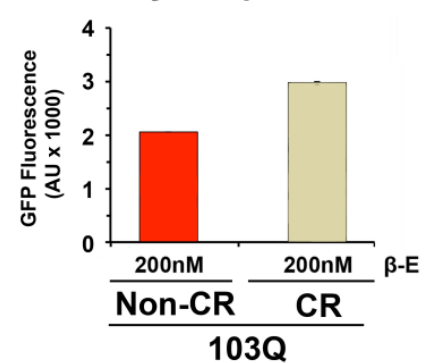

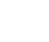

.

(1)


growing in non-CR medium (Figure 4B).

Because mitochondrial respiration is not required for CLS in CR-treated cells, our data indicate that 1030 toxicity during CLS involves multiorganelle pathways as it does in growing cells. As for the anti-toxicity interventions discussed in previous paragraphs, the CR-treatment effect was not due to decreases in polyQ protein, since, like for cells grown in WOEG, polyQ expression was estimated to be significantly higher in CR cells than those grown in standard glucose-containing media (Figure 4C).
Mitochondrial respiration is essential for suppression of PolyQ toxicity via enhanced mitochondrial biogenesis

Although all the anti-103Q toxicity interventions presented in the previous sections involve increases in cellular respiration during growth, we wanted to further explore whether the mitochondrial contribution to this protection involves OXPHOS or if it may relate to some other pathway affected by enhancement of mitochondrial biogenesis. In order to test whether mitochondrial respiration is required for HAP4-mediated suppression of $103 Q$ proteotoxicity, we

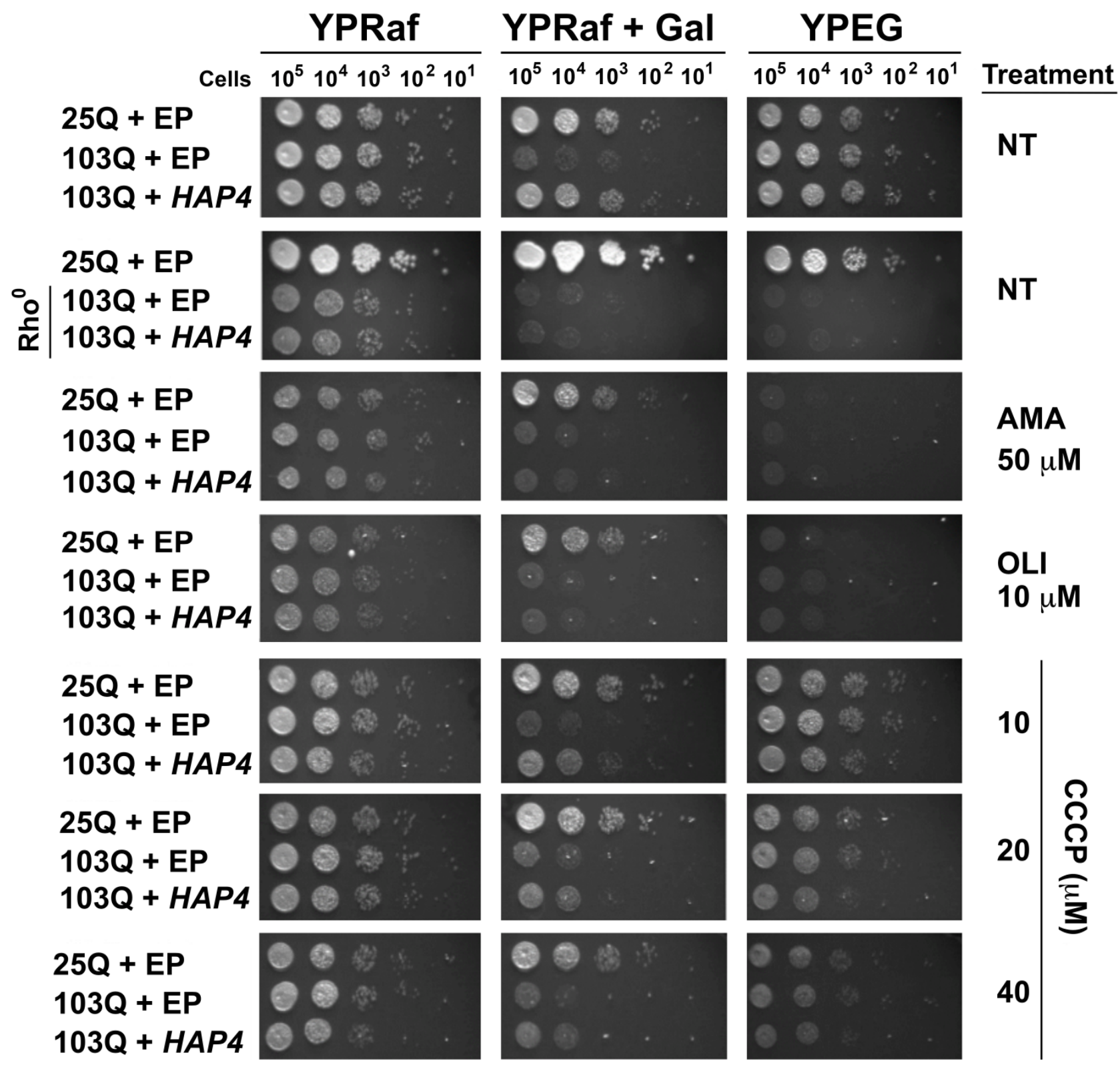

FIGURE 5: Mitochondrial respiration is essential for suppression of polyQ-induced toxicity by HAP4 overexpression. Serial dilutions of yeast strains on non-inducing media (YPRaf), polyQ-inducing media (YPRaf+Gal), and respiratory media (YPEG) after 2 days of growth at $30^{\circ} \mathrm{C}$. Rho ${ }^{\circ}$ indicates cells without mitochondrial DNA. EP, Empty plasmid; NT, no treatment (NT); AMA, antimycin A; CCCP, Carbonyl cyanide mchlorophenyl hydrazone; OLI, oligomycin. 
turned to our mitotic models of galactose-induced polyQ expression during exponential growth. For these assays we used strains expressing either non-toxic $25 \mathrm{Q}$ or toxic $103 \mathrm{Q}$ motifs further modified to overexpress HAP4 or to carry an empty episomal plasmid (EP). Here, we abolished mitochondrial OXPHOS function by genetic and pharmacologic manipulations and determined the efficacy of HAP4-mediated suppression of 103Q-induced cytotoxicities.

First, we used previously reported $103 Q$ yeast, with or without the overexpression of HAP4, that had been completely depleted of mitochondrial DNA (mtDNA) and therefore are rho $^{0}$ strains [31]. The mtDNA encodes essential components of the OXPHOS system, and, therefore, rho $^{\circ}$ yeast are unable to grow in complete media in the presence of respiratory substrates such as ethanol and glycerol (YPEG). Because rho ${ }^{\circ}$ cells do not grow efficiently in galactose-containing media, we used the nonrepressible raffinose as the fermentable carbon source and induced protein expression when required by supplementing the plates with $0.25 \%$ galactose. As shown in Figure 5, HAP4 OE suppressed 103Q-induced toxicity, as previously reported [4]. As expected, the suppression was only effective in rho ${ }^{+}$strains, containing mtDNA, but not in rho ${ }^{0}$ strains, suggesting that HAP4-mediated suppression requires functional mitochondrial respiration.

In addition to the genetic approach, we also pharmacologically inhibited mitochondrial respiratory complex III activity with antimycin A (AMA) that prevents growth in respiratory media (YPEG). AMA treatment abolished the suppression by HAP4 OE (Figure 5). Similar outcomes were obtained when mitochondrial OXPHOS was impaired by supplementation of the growth media with oligomycin (OLI), an inhibitor of the $F_{1} F_{0}$ ATPase. We next treated the cells with increasing concentrations of the ionophore carbonyl cyanide m-chlorophenyl hydrazine (CCCP). CCCP causes an uncoupling of the mitochondrial proton gradient from ATP production, causing a futile increase in mitochondrial respiration. Although still effective, a reduction in the protective effects of HAP4 OE in 103Qexpressing cells was seen with increasing concentrations of CCCP (Figure 5). These results strongly indicate that coupled mitochondrial respiration is the essential component of the HAP4 mechanism of protection against proteotoxicity in yeast.

Attenuation of PolyQ-induced neurodegeneration in a fly model of Huntington's disease via enhanced mitochondrial biogenesis

To test whether our results in yeast could be replicated in higher organisms, we turned to a Drosophila model of HD. Similar to the function of the Hap2/3/4/5 complex in yeast, PGC-1 $\alpha$ in higher organisms is a transcriptional activator critical in regulating mitochondrial biogenesis [25]. In Drosophila, overexpression of the PGC-1 $\alpha$ homolog (DmPGC$1 /$ spargel) has been shown to be sufficient to increase mitochondrial OXPHOS activity, which leads to an extension of fly life span and improvement in tissue homeostasis in aged flies [32]. Here, we used a pan-neuronal driver elav ${ }^{\text {C155-GAL4 }}$ to express the exon 1 of human huntingtin gene with pathological 93 polyQ repeats ( $\mathrm{hHttExQ93)}$ or nonpathological 20 polyQ repeats (hHttExQ20) in the fly nervous system [33]. First, we tested the locomotor activity of the flies by a negative geotaxis assay. As shown in Figure 6, while flies expressing hHttExQ20 showed similar climbing behavior as wild-type flies $(y w)$, expression of hHttExQ93 caused an age-dependent decline of the climbing performance that was significant at 10 and 20 days after eclosion

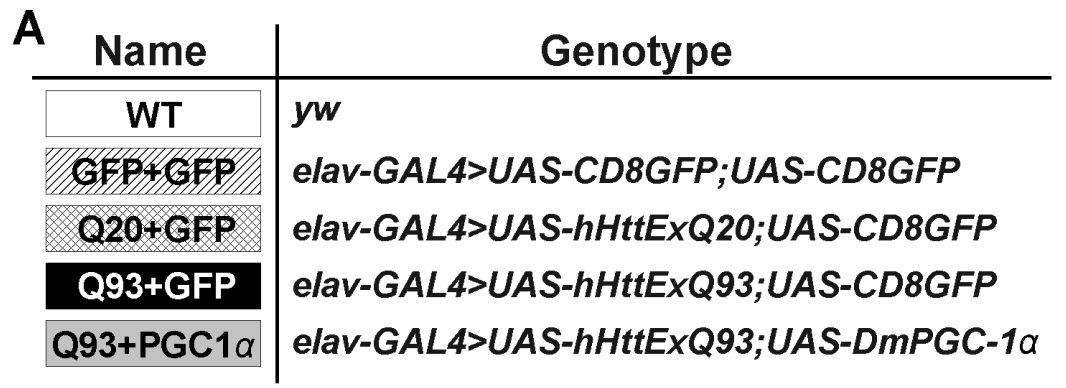

B

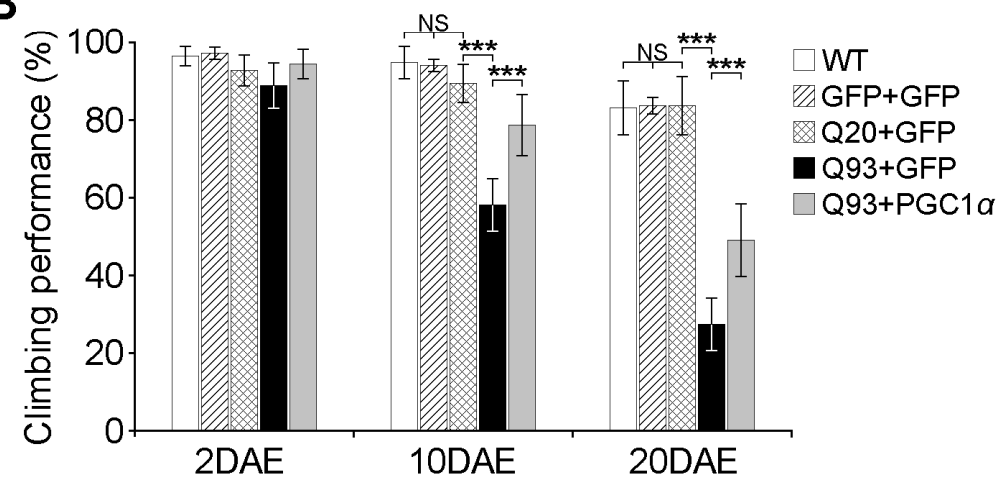

FIGURE 6: DmPGC-1 $\alpha /$ spargel protects against hHttExQ93-induced climbing defects. (A) List of genotypes of the transgenic flies examined. The first exon of human huntingtin gene with 20 (UAS-hHttExQ20) or 93 (UAS-hHttExQ93) repeats of polyQ, and GFP (UAS-CD8GFP) or DmPGC-1 $\alpha$ /spargel (UAS-DmPGC-1 $\alpha$ ) were expressed by the pan-neuronal driver elav ${ }^{\mathrm{C} 155-}$ ${ }^{G A L 4}$. (B) Climbing performance of wild-type, GFP over-expressing flies, and hHtt-expressing flies that co-expressed GFP or PCG-1 $\alpha$ at the ages of 2, 10 and 20 DAE (days after eclosion). Ten groups (10 flies in each, total one hundred flies) of each genotype and age were tested. All data were presented as mean \pm S.D. $n=10$. Significance level was established by One-Way ANOVA post hoc Tukey's test. ${ }^{* * *} \mathrm{P}<0.001$. 
(DAE). This decline of climbing performance was suppressed by co-expression of DmPGC-1 $\alpha$. Consistent with a previous report [32], overexpression of DmPGC$1 \alpha$ enhanced mitochondrial biogenesis by $50 \%$ in fly brains, as monitored by the steady state levels of ATP $5 \alpha$, a subunit of mitochondrial $F_{1} F_{0}$-ATP synthase [34, 35] (Figure 7A-B).

To examine the cellular effect of enhancing mitochondria biogenesis on mutant $\mathrm{Htt}$-induced neurodegeneration, we determined the level of caspase-3 activation, a hallmark of apoptosis [36], at early (2 DAE) and late (20 DAE) ages. Neuronal expression of hHttExQ93 induced an increased level of cleaved caspase- 3 throughout the entire fly brain at 2 DAE, which could be suppressed by coexpression of DmPGC-1 $\alpha$ (Figure 7C). Therefore, neuronal apoptosis occurred early following hHttExQ93 expression, before the onset of significant decline in climbing behavior. To further quantitatively monitor the activation of apoptosis, we measured the expression level of caspase 3 P12, the activated and fully processed product of pro-caspase 3 [37] Consistently, expression of hHttExQ93 significantly increased $\mathrm{P} 12$ level in the fly brains, which could be attenuated by overexpression of DmPGC-1 $\alpha$ at both 2 DAE and 20 DAE (Figure 7D-E).

Collectively, these results indicate that enhancing mito- chondrial biogenesis by overexpressing DmPGC-1 $\alpha$ protects against polyQ-induced neurotoxicity, and this neuroprotective mechanism is likely conserved between yeast and flies.

\section{DISCUSSION}

The role of mitochondrial respiration and oxidative phosphorylation in proteotoxic neurodegeneration is a longstanding question, and the mechanism by which enhancement of mitochondrial biogenesis protects against proteotoxicity is still poorly understood. Polyglutamine toxicity is pleiotropic, affecting multiple pathways and organelles, including mitochondria. Among the mitochondrial pathways, our studies using yeast CLS and fly models of neurodegenerative proteopathies identify OXPHOS as the pathway that is most affected by polyglutamine expression and oligomerization. Notably, we reveal that genetic and nutritional interventions that enhance mitochondrial biognesis to precondition cells and tissues with high respiration serve to attenuate polyQinduced proteotoxicity.

Most previous studies of proteotoxicity suppression in yeast models have used rapidly dividing mitotic cells, and have established acute toxicity with a high expression of
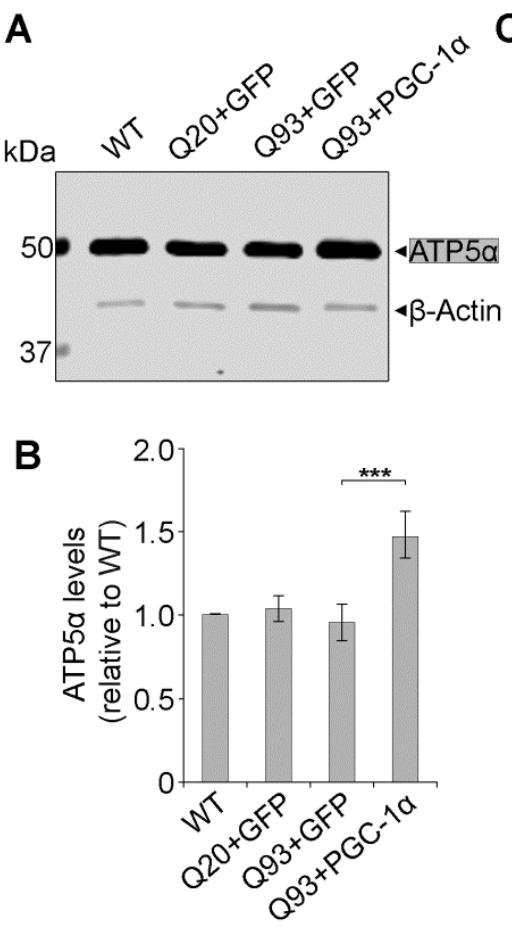

C
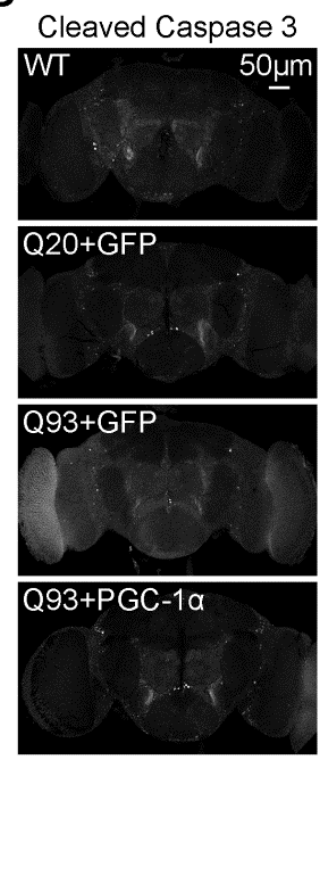

D
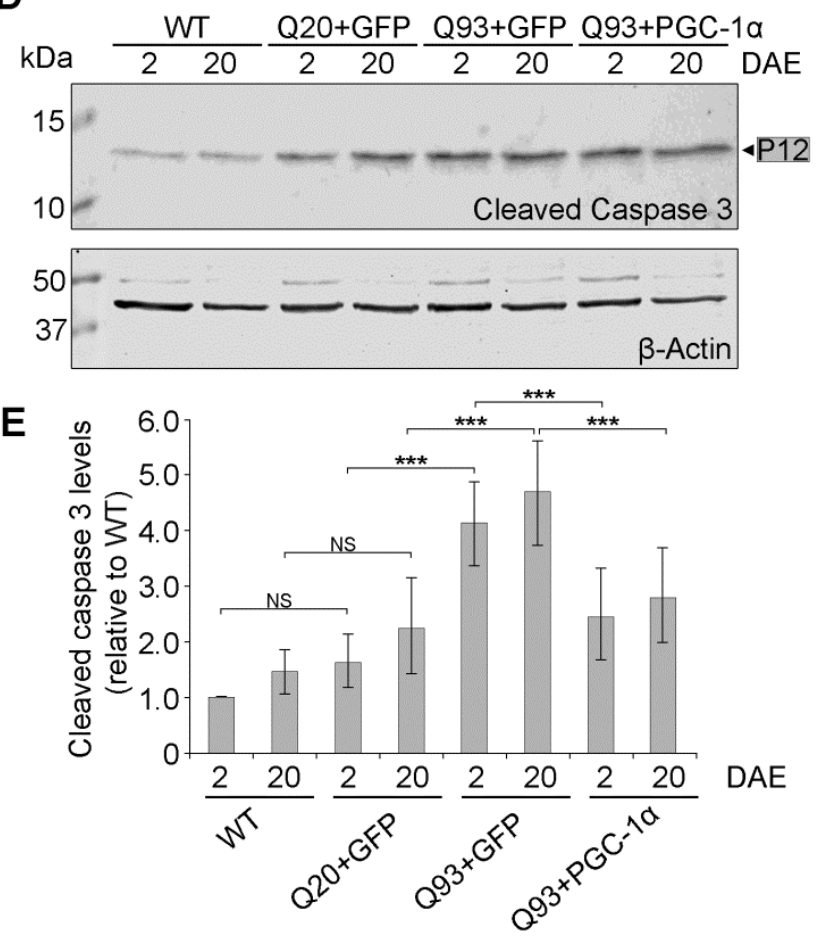

FIGURE 7: DmPGC-1 $\alpha$ /spargel suppresses hHttExQ93-induced activation of cell apoptosis. (A-B) Western blot analysis (A) and quantification (B) of proteins extracted from 2 DAE wild-type, hHttExQ20- or hHttExQ93-overexpressing fly heads. ATP5 $\alpha$ was used as a mitochondrial marker. $\beta$-Actin was used as an internal control. For quantification, fold change relative to the level of WT group is displayed. (C) Adult (2 DAE) female brains of wild-type, hHttExQ20- or hHttExQ93-overexpressing flies were probed for cleaved caspase-3. Scale bar is $50 \mu \mathrm{m}$. (D-E) Western blot analysis (D) and quantification (E) of proteins extracted from 2 DAE and 20 DAE wild-type, hHttExQ20- or hHttExQ93overexpressing fly heads. For quantification, P12 was considered as cleaved caspase-3 (grey box): -fold change relative to each level in 2 DAE wild-type flies. $\beta$-Actin was used as an internal control. All data were presented as mean \pm S.D., $n=3$. Significance level was established by One-Way ANOVA post hoc Bonferroni test. ${ }^{* * *} \mathrm{P}<0.001$. 
toxic proteins using galactose-inducible promoters [4-7, 38]. Here, using a $\beta$-estadiol expression system capable of inducing toxic polyQ expression at the point of transition to stationary phase [17], we have documented an early mitochondrial respiratory defect. We have also shown that enhancement of mitochondrial biogenesis by overexpression of HAP4 partially suppresses polyQ-induced toxicity in the stationary phase of the CLS assay, a model for neuronal aging, as previosuly reported for the polyQinduced growth deficit in the exponential phase [4]. Importantly, we report that, in the exponential growth model, anti-polyQ protection by HAP4 overexpression is abolished with the inhibiton of mitochondrial respiration, even though the increase in mitochondrial biogenesis remains, thus demonstrating that protecting OXPHOS pathway is a key for polyQ toxicity attenuation. We have further exploited the yeast paradigm to demonstrate that growth in non-fermentable media and CR media, nutritional interventions known to increase mitochondrial biogenesis and mitochondrial respiration during growth, also confer protection against stationary-phase polyQ toxicity. In the case of $\mathrm{CR}$, multiple additional pathways could be relevant. For example, despite the consensus that mutant polyQ- and alpha-synuclein-induced toxicities involve several distinct pathways, it has been recently reported that $C R$ reduces alpha-synuclein toxicity in aged yeast cells by controlling the maintenance of autophagic activity at homeostatic levels [39]. The possibility exist, however, that the control of autophagy in these growth conditions is a consequence of the CR-induced enhanced OXPHOS function.

Over the several experimental settings presented here, we have observed that enhanced respiration during growth results in lowered endogenous cell respiration during stationary phase, the most extreme case being CR-treated cells, which survive without utilizing respiratory metabolism [16]. We have previously shown that higher respiration during growth is associated with higher ROS generation, which can act as signaling molecules to induce an adaptive response in stationary phase involving metabolic remodeling and slow metabolism. This is particularly true for cells grown under CR conditions or in the presence of rapamycin to inhibit the TOR pathway [16, 40], and it is also true for cells overexpressing HAP4 [16].

We need to emphasize, however, that we performed our respiratory assays using cells in their culture media, and therefore we are measuring "real time" respiration. CR-treated cells maintain a high mitochondrial mass in stationary phase, but this capacity is not utilized for respiration during CLS. The relationship between high respiration during growth phases and chronological life span is also intriguing. An important example are the puf3 mutant strains, which include an increased OXPHOS abundance and respiration during all phases of growth, but a wild-type CLS [41]. In agreement with this, we have shown that increased respiration during growth (e.g., by HAP4 OE) is not sufficient to extend CLS of wild-type cells if it is not accompanied by an enhancement of cell protection systems that promote survival in the stationary phase as for a tor1 mutant- and CR-treated cells [16]. However, in chronologically aged 103Q-expressing cells, in which mitochondrial function is altered, even HAP4 overexpression is able to provide the enhanced mitochondrial mass buffer needed to overcome the polyQinduced mitochondrial toxicity.

As an important validation of the observations made in the yeast models, we have confirmed our HAP4 overexpression results in a fly model of $H D$, in which we have shown that overexpression of the Drosophila PGC-1 $\alpha$ homolog (DmPGC-1/spargel) is also able to protect against the behavioral deficits and neurodegeneration induced by polyQ proteins. It is important to note the potential difference in hHttExQ93 expression levels between our study and previous studies using the same transgenic line [42]. In our study, to ensure similar expression level of hHttExQ93 among all groups, we used UAS-GFP as a control transgenic element. Therefore, the level of hHttExQ93 expression in our study is likely lower than that in previous studies. As a result, the progression of degeneration is slower as hHttExQ93-expressing flies in our study all survived beyond $20 \mathrm{DAE}$, while a median lifespan of 10 DAE (maximum 17 DAE) was reported in the previous study [42]. The slightly slower degeneration gave us the temporal resolution to assess the age-dependent decline in climbing behavior and the suppression by PGC-1 $\alpha$. Interestingly, we observed the activation of apoptosis before the onset of behavior defects, suggesting apoptosis as an early event and a likely cause of degeneration. Importantly, PGC-1 $\alpha$ expression suppresses apoptosis from the early age, providing the molecular basis for the neuroprotection offered by PGC-1 $\alpha$.

These results are in agreement with previous studies showing that transcriptional repression of PGC-1 $\alpha$ by mutant $\mathrm{Htt}$ leads to mitochondrial dysfunction and neurodegeneration in HD mouse models and that exogenous expression of PGC- $1 \alpha$ in this model protects against mutant $\mathrm{Htt}$-induced neurotoxicity $[3,43]$. Furthermore, PGC-1 $\alpha$ has been proposed to be a modifier of onset age in HD patients, although functional studies are needed to confirm this and to identify the genetic variations in PGC-1 $\alpha$ that enhance or alleviate HD pathogenesis [44-46].

In conclusion, our results in post-mitotic yeast, using the CLS assay, as well as our results in the Drosophila model of HD, shed new light on how mitochondrial respiration and OXPHOS-dependent pathways are affected by polyQ-induced toxicity, and support the need for further research into the possibile therapeutic potential of interventions aimed at increasing mitochondrial biogenesis in patients suffering from HD and other neurodegenerative proteopathies.

\section{MATERIALS AND METHODS \\ Yeast strains and methods}

Yeast strain and media

The yeast strains used in this study were constructed from the previously reported $25 Q$ and $103 Q$ isogeneic strains, and the respective HAP4 overexpressing strains, with the $S$. cerevisiae W303-1A background $[4,5]$, modified with the $\beta$-estradiolinducible TEF1-7 promoter described in [17]. Compositions of 
the different growth media have been described [47-49]. Prior to induction with galactose or the indicated amount of $\beta$ estradiol, all strains were grown in non-inducible media. For the chronological lifespan assay, strains were grown in the indicated media, using the methods described in [50].

\section{Yeast chronological life span determinations}

Most chronological life span determinations were performed in cells grown in liquid synthetic complete media containing $2 \%$ glucose (WOGLU) supplemented with standard amounts of amino acids and nucleotide bases as previously described including a four fold excess of the supplements tryptophan, leucine, histidine, methionine, adenine and uracyl to avoid possible artifacts due to the auxotrophic deficiencies of the strains [50]. For growth in respiratory media, glucose was substituted by EG ( $2 \%$ ethanol and $2 \%$ glycerol). For calorie restriction (CR) experiments, glucose concentration was reduced to $0.5 \%$. Briefly, yeast strains from frozen stock $\left(-80^{\circ} \mathrm{C}\right)$ were patched onto complete YPD ( $2 \%$ glucose) or YPEG $(2 \%$ ethanol and $2 \%$ glycerol) agar plates and incubated at $30^{\circ} \mathrm{C}$. The following day, cells were inoculated into $10 \mathrm{ml}$ of minimum WO media with the appropriate carbon sources and grown overnight. After 24 hours, cells were inoculated into 50 $\mathrm{ml}$ of WO media in 250-ml flasks to an optical density at 600 $\mathrm{nm}\left(\mathrm{OD}^{600}\right)$ of 0.250 . Cultures were grown with shaking (250 $\mathrm{rpm}$ ) at $30^{\circ} \mathrm{C}$. Maximum cell density is normally reached after 72 hours of growth in WO, therefore 3 days after inoculation was considered as "day 0 " of chronological life span. Subsequently, cellular viability was determined at the indicated days by either propidium iodide (PI) staining followed by flow cytometry analysis or by the colony formation unit (CFU) assay.

\section{Propidium iodide staining and flow cytometry analysis}

Membrane integrity as a marker of cell viability was assessed by PI staining (Molecular Probes, Eugene, OR, USA) and analyzed by flow cytometry as previously described [50]. Every other day, a sample of stationary phase cultures containing $10^{6}$ yeast cells per $\mathrm{ml}$ in phosphate-buffered saline (PBS) was incubated for $30 \mathrm{~min}$ at $30^{\circ} \mathrm{C}$ in the presence of $2 \mu \mathrm{M}$ PI. Flow cytometry analysis was performed on a Becton Dickinson (BD) LSRFortessa $^{\mathrm{TM}}$ cell analyzer as described [50]. Excitation was performed using a yellow/green laser at $561 \mathrm{~nm}$; emission was detected using a $20 \mathrm{~nm}$ bandpass filter centered at $660 \mathrm{~nm}$ (Becton Dickinson, NJ, USA). For each yeast population, three samples of ten thousand cells were analyzed.

\section{Colony formation unit assay}

To perform the colony formation unit (CFU) assay, cell number was estimated by optical density (OD) for each population and serial dilutions of different cultures were plated onto 3 or 4 YPD plates at an approximate concentration of 100 cells per plate. Plates were incubated at $30^{\circ} \mathrm{C}$ for $48 \mathrm{~h}$ and $\mathrm{CFU}$ were counted.

\section{Fluorescence microscopy}

Wide-field fluorescence microscopy for verification of polyQGFP expression after $\beta$-estradiol induction in yeast was performed as described [4]. Briefly, we used an Olympus fluorescence BX61 microscope equipped with Nomarski differential interference contrast (DIC) optics, a Uplan Apo 100x objective (NA 1.35), a Roper CoolSnap HQ camera, and Sutter Lambda10-2 excitation and emission filter wheels, and a $175 \mathrm{~W}$
Xenon remote source with liquid light guide. Briefly, PI-stained cells were mounted on to slides and examined using a CY3 filter. Images were acquired using SlideBook 4.01 (Intelligent Imaging Innovations, Denver, CO, USA).

\section{Cell respiration}

Assessment of yeast endogenous cellular respiration was performed polarographically using a Clark-type oxygen electrode (Hansatech Instruments, Norfolk, UK) at $30^{\circ} \mathrm{C}$ as described [51]. Measurement of respiration in the exponential phase of growth was performed $16 \mathrm{~h}$ after concomitant strain inoculation and induction. For exponential phase respiration, cells were grown overnight in the indicated media, reinoculated in fresh media at the same confluence $\left(O D^{600}=0.250\right)$ and grown for 3-6 $\mathrm{h}$, as indicated, to measure maximal cellular respiration. For stationary phase respiration, cell were treated as describe above and grown for $72 \mathrm{~h}$ (day 0 ) before measure respiration, to allow cell to reach stationary phase. The specific activities reported were corrected for $\mathrm{KCN}$-insensitive respiration.

\section{Chemical treatments}

Serial dilution growth tests were performed in solid media containing glucose, raffinose or galactose as the carbon source, supplemented or not with several mitochondrial poisons. Plates were supplemented with either $50 \mu \mathrm{M}$ antimycin A (Sigma) to inhibit the mitochondrial respiratory chain complex III or $10 \mu \mathrm{M}$ oligomycin (Sigma) to inhibit the mitochondrial $\mathrm{F}_{1} \mathrm{~F}_{0}$ ATP synthase. Plates were also supplemented with increasing concentrations (10-40 $\mu \mathrm{M})$ of the uncoupler CCCP (carbonyl cyanide m-chlorophenyl hydrazine). In the "untreated" plates, an equal volume of drug vehicle (ethanol) was added as a control.

\section{Drosophila strains and methods}

Drosophila strains and culture conditions

All fly strains were maintained on a cornmeal-molasses-yeast medium at room temperature $\left(\sim 22^{\circ} \mathrm{C}\right)$ with $60-65 \%$ humidity. The following Drosophila strains were used in the studies: elav ${ }^{\text {C155-GAL4 }}$ and UAS-CD8GFP were obtained from Bloomington Drosophila Stock Center. UAS-hHttExQ2O and UAShHttExQ93 were obtained from Dr. Leslie Thompson [33]. UAS-DmPGC-1 $\alpha$ (UAS-srl) was obtained from Dr. Christian Frei [52].

\section{Negative geotaxis behavior assay}

Ten age-matched female flies from each genotype were placed in a vial marked with a circle $8 \mathrm{~cm}$ above the bottom surface. The flies were gently tapped to the bottom and given $10 \mathrm{~s}$ to climb. After $10 \mathrm{~s}$, the number of flies that successfully climbed above the $8 \mathrm{~cm}$ mark was recorded and divided by the total number of flies. The assay was repeated 10 times and the data were represented as the averaged percentages. Ten independent groups (total 100 flies) per genotype was tested [53].

\section{Protein extraction and western blot analysis}

Proteins were extracted from fly heads using the homogenizing buffer containing $10 \mathrm{mM}$ HEPES pH 7.9, $1.5 \mathrm{mM} \mathrm{MgCl}_{2}, 10$ $\mathrm{mM} \mathrm{KCl}, 1 \mathrm{mM}$ dithiothreitol (DTT) and $0.5 \mathrm{mM}$ phenylmethylsulphonyl fluoride (PMSF). Samples were then spun at $14,000 \mathrm{~g}$ for $30 \mathrm{~min}$ at $4^{\circ} \mathrm{C}$ and the supernatant was collected as supernatant 1 . The pellets were resuspended with the protein ex- 
traction buffer containing $30 \mathrm{mM}$ HEPES $\mathrm{pH} 7.9,0.6 \mathrm{M} \mathrm{NaCl}$, $1.5 \mathrm{mM} \mathrm{MgCl} 2,0.4 \mathrm{mM}$ EDTA, $1 \mathrm{mM}$ DTT, 25\% glycerol, 1\% NP40 and $0.5 \mathrm{mM}$ PMSF and incubated at $4^{\circ} \mathrm{C}$ for $30 \mathrm{~min}$ with vortexing every $6 \mathrm{~min}$. Then the supernatant 2 was collected after $30 \mathrm{~min}$ centrifugation at $14,000 \mathrm{~g}$ at $4^{\circ} \mathrm{C}$. Next, supernatants 1 and 2 were equally mixed and heated at $95^{\circ} \mathrm{C}$ with $4 \times$ Laemmli sample buffer [54]. Samples were loaded onto $15 \%$ SDS-polyacrylamide gels and transferred to nitrocellulose membranes. Blots were probed with anti-ATP5 $\alpha$ antibody (1:10,000, Abcam), anti-cleaved caspase 3 (1:1,000, Cell Signaling), or anti- $\beta$-Actin (1:10,000, Sigma) primary antibodies, and then probed with infrared dye-conjugated IR700 and IR800 secondary antibodies (1:10,000, LI-COR Biosciences). Blots were imaged and processed on an Odyssey Infrared Imaging system.

\section{Immunohistochemistry of fly brains}

Adult brains were dissected in ice cold PBS (pH 7.4), fixed in $4 \%$ formaldehyde for $15 \mathrm{~min}$ and washed in PBS with $0.4 \%$ Triton X-100 (PBTX). Brains were incubated at $4^{\circ} \mathrm{C}$ overnight with the following antibodies diluted in PBTX with $5 \%$ normal goat serum: anti-cleaved caspase 3 antibody (Asp175; 1:250, Cell Signaling); secondary antibody conjugated to Alexa 488 (1:250, Jackson ImmunoResearch, Molecular Probes). Tissues were then mounted on microscope slides in Vectashield Mounting Medium for Fluorescence (Vector Laboratories).

\section{Confocal image acquisition and processing}

Confocal microscopy was performed with an Olympus IX81 confocal microscope using an Olympus PlanApo N 20x objective. Images from different genotypes were captured using the same scan setting and parameters. Images were processed using FluoView 10-ASW (Olympus) and assembled using Adobe Photoshop CS6 (Adobe Systems).

\section{REFERENCES}

1. Lin MT, Beal MF (2006). Mitochondrial dysfunction and oxidative stress in neurodegenerative diseases. Nature 443: 787-795.

2. Panov AV, Gutekunst CA, Leavitt BR, Hayden MR, Burke JR, Strittmatter WJ, Greenamyre JT (2002). Early mitochondrial calcium defects in Huntington's disease are a direct effect of polyglutamines. Nat. Neurosci. 5: 731-736.

3. Cui L, Jeong H, Borovecki F, Parkhurst CN, Tanese N, Krainc D (2006) Transcriptional repression of PGC-1alpha by mutant huntingtin leads to mitochondrial dysfunction and neurodegeneration. Cell 127: 59-69.

4. Ocampo A, Zambrano A, Barrientos A (2010). Suppression of polyglutamine-induced cytotoxicity in Saccharomyces cerevisiae by enhancement of mitochondrial biogenesis. FASEB J. 24: 1431-1441.

5. Solans A, Zambrano A, Rodriguez M, Barrientos A (2006). Cytotoxicity of a mutant huntingtin fragment in yeast involves early alterations in mitochondrial OXPHOS complexes II and III. Hum. Mol. Genet. 15: 3063-3081.

6. Meriin AB, Zhang $X$, He X, Newnam GP, Chernoff YO, Sherman MY (2002). Huntington toxicity in yeast model depends on polyglutamine aggregation mediated by a prion-like protein Rnq1. J. Cell Biol. 157: 997-1004.

7. Willingham S, Outeiro TF, DeVit MJ, Lindquist SL, Muchowski PJ (2003). Yeast genes that enhance the toxicity of a mutant huntingtin fragment or alpha-synuclein. Science 302: 1769-1772.

\section{Statistical Analysis}

All experimental measurements were done at least in triplicate. All results are presented as means \pm S.E.M. or S.D. as appropriate. Significance testing was performed using the Student's T-test or One-Way ANOVA, as noted in the figure legends.

\section{ACKNOWLEDGMENTS}

This work is supported by grants from the National Institutes of Health (NIH) R01 GM071775, GM105781 and GM112179 (to A.B.) and 2R56NS064269 (to R.G.Z), and of the US Department of the Army (ARO \#\#65594-LS to A.B.).

\section{SUPPLEMENTAL MATERIAL}

All supplemental data for this article are available online at www.microbialcell.com.

\section{CONFLICT OF INTEREST}

The authors declare no conflict of interest.

\section{COPYRIGHT}

(C) 2016 Ruetenik et al. This is an open-access article released under the terms of the Creative Commons Attribution (CC BY) license, which allows the unrestricted use, distribution, and reproduction in any medium, provided the original author and source are acknowledged.

Please cite this article as: Andrea L. Ruetenik, Alejandro Ocampo, Kai Ruan, Yi Zhu, Chong Li, R. Grace Zhai and Antoni Barrientos (2016). Attenuation of polyglutamine-induced toxicity by enhancement of mitochondrial OXPHOS in yeast and fly models of aging. Microbial Cell 3(8): 338-351. doi: 10.15698/mic2016.08.518

8. Braun RJ, Buttner S, Ring J, Kroemer G, Madeo F (2009). Nervous yeast: modeling neurotoxic cell death. Trends Biochem. Sci. 35: 135144.

9. Outeiro TF, Giorgini F (2006). Yeast as a drug discovery platform in Huntington's and Parkinson's diseases. Biotechnol. J. 1: 258-269.

10. Krobitsch S, Lindquist S (2000). Aggregation of huntingtin in yeast varies with the length of the polyglutamine expansion and the expression of chaperone proteins. Proc. Natl. Acad. Sci. USA 97: 15891594.

11. Duennwald ML, Lindquist S (2008). Impaired ERAD and ER stress are early and specific events in polyglutamine toxicity. Genes Dev. 22: 3308-3319.

12. Forsburg SL, Guarente $L$ (1989). Identification and characterization of HAP4: a third component of the CCAAT-bound HAP2/HAP3 heteromer. Genes Dev. 3: 1166-1178.

13. Buttner $S$, Bitto A, Ring J, Augsten $M$, Zabrocki $P$, Eisenberg $T$, Jungwirth $\mathrm{H}$, Hutter S, Carmona-Gutierrez D, Kroemer G, Winderickx J, Madeo $F$ (2008). Functional mitochondria are required for alphasynuclein toxicity in aging yeast. J. Biol. Chem. 283: 7554-7560.

14. Longo VD, Shadel GS, Kaeberlein M, Kennedy B (2012). Replicative and Chronological Aging in Saccharomyces cerevisiae. Cell 16: 18-31. 
15. Smith DL, Jr., McClure JM, Matecic M, Smith JS (2007). Calorie restriction extends the chronological lifespan of Saccharomyces cerevisiae independently of the Sirtuins. Aging Cell 6: 649-662.

16. Ocampo A, Liu J, Schroeder EA, Shadel GS, Barrientos A (2012). Mitochondrial respiratory thresholds regulate yeast chronological life span and its extension by caloric restriction. Cell Metab. 16: 55-67.

17. Ocampo A, Barrientos A (2008). From the bakery to the brain business: developing inducible yeast models of human neurodegenerative disorders. Biotechniques 45: Pvii-xiv.

18. Louvion JF, Havaux-Copf B, Picard D (1993). Fusion of GAL4-VP16 to a steroid-binding domain provides a tool for gratuitous induction of galactose-responsive genes in yeast. Gene 131: 129-134.

19. Piper PW, Harris NL, MacLean M (2006). Preadaptation to efficient respiratory maintenance is essential both for maximal longevity and the retention of replicative potential in chronologically ageing yeast. Mech. Ageing Dev. 127: 733-740.

20. Barros MH, Bandy B, Tahara EB, Kowaltowski AJ (2004). Higher respiratory activity decreases mitochondrial reactive oxygen release and increases life span in Saccharomyces cerevisiae. J. Biol. Chem. 279: 49883-49888.

21. Burtner CR, Murakami CJ, Kennedy BK, Kaeberlein M (2009). A molecular mechanism of chronological aging in yeast. Cell Cycle 8: 1256-1270.

22. Matecic M, Smith DL, Pan X, Maqani N, Bekiranov S, Boeke JD, Smith JS (2010). A microarray-based genetic screen for yeast chronological aging factors. PLoS Genet. 6: e1000921.

23. Wei M, Fabrizio P, Hu J, Ge H, Cheng C, Li L, Longo VD (2008). Life span extension by calorie restriction depends on $\operatorname{Rim} 15$ and transcription factors downstream of Ras/PKA, Tor, and Sch9. PLoS Genet. 4: e13.

24. Oliveira GA, Tahara EB, Gombert AK, Barros MH, Kowaltowski AJ (2008). Increased aerobic metabolism is essential for the beneficial effects of caloric restriction on yeast life span. J. Bioenerg. Biomembr. 40: $381-388$

25. Ruetenik A, Barrientos A (2015). Dietary restriction, mitochondrial function and aging: from yeast to humans. Biochim. Biophys. Acta 12: 00086-00089.

26. Carlson M (1999). Glucose repression in yeast. Curr. Opin. Microbiol. 2: 202-207.

27. Brauer MJ, Saldanha AJ, Dolinski K, Botstein D (2005). Homeostatic adjustment and metabolic remodeling in glucose-limited yeast cultures. Mol. Biol. Cell. 16: 2503-2517.

28. Roberts GG, Hudson AP (2006). Transcriptome profiling of Saccharomyces cerevisiae during a transition from fermentative to glycerol-based respiratory growth reveals extensive metabolic and structural remodeling. Mol. Genet. Genomics. 276: 170-186.

29. Werner-Washburne M, Braun EL, Crawford ME, Peck VM (1996). Stationary phase in Saccharomyces cerevisiae. Mol. Microbiol. 19: 1159-1166.

30. Goldberg AA, Bourque SD, Kyryakov P, Gregg C, Boukh-Viner T, Beach A, Burstein MT, Machkalyan G, Richard V, Rampersad S, Cyr D, Milijevic S, Titorenko VI (2009). Effect of calorie restriction on the metabolic history of chronologically aging yeast. Exp. Gerontol. 44: 555-571.

31. Ocampo A, Liu J, Barrientos A (2013). NAD+ salvage pathway proteins suppress proteotoxicity in yeast models of neurodegeneration by promoting the clearance of misfolded/oligomerized proteins. Hum. Mol. Genet. 22: 1699-1708.
32. Rera $M$, Bahadorani $S$, Cho J, Koehler $C L$, Ulgherait $M$, Hur JH, Ansari WS, Lo T, Jr., Jones DL, Walker DW (2011). Modulation of longevity and tissue homeostasis by the Drosophila PGC-1 homolog. Cell Metab. 14: 623-634.

33. Steffan JS, Bodai L, Pallos J, Poelman M, McCampbell A, Apostol BL, Kazantsev A, Schmidt E, Zhu YZ, Greenwald M, Kurokawa R, Housman DE, Jackson GR, Marsh JL, Thompson LM (2001). Histone deacetylase inhibitors arrest polyglutamine-dependent neurodegeneration in Drosophila. Nature 413: 739-743.

34. Ivatt RM, Sanchez-Martinez A, Godena VK, Brown S, Ziviani E, Whitworth AJ (2014). Genome-wide RNAi screen identifies the Parkinson disease GWAS risk locus SREBF1 as a regulator of mitophagy. Proc. Natl. Acad. Sci. USA 111: 8494-8499.

35. Chen CL, Hu Y, Udeshi ND, Lau TY, Wirtz-Peitz F, He L, Ting AY, Carr $\mathrm{SA}$, Perrimon N (2015). Proteomic mapping in live Drosophila tissues using an engineered ascorbate peroxidase. Proc. Natl. Acad. Sci. USA 112: $12093-12098$.

36. Elmore S (2007). Apoptosis: a review of programmed cell death. Toxicol. Pathol. 35: 495-516.

37. Han Z, Hendrickson EA, Bremner TA, Wyche JH (1997). A sequential two-step mechanism for the production of the mature p17:p12 form of caspase-3 in vitro. J. Biol. Chem. 272: 13432-13436.

38. Outeiro TF, Lindquist S (2003). Yeast cells provide insight into alpha-synuclein biology and pathobiology. Science 302: 1772-1775.

39. Guedes A, Ludovico P, Sampaio-Marques B (2016). Caloric restriction alleviates alpha-synuclein toxicity in aged yeast cells by controlling the opposite roles of Tor1 and Sir2 on autophagy. Mech. Ageing Dev. 21: 30048-30043.

40. Pan Y, Schroeder EA, Ocampo A, Barrientos A, Shadel GS (2011). Regulation of yeast chronological life span by TORC1 via adaptive mitochondrial ROS signaling. Cell Metab. 13: 668-678.

41. Chatenay-Lapointe M, Shadel GS (2011). Repression of mitochondrial translation, respiration and a metabolic cycle-regulated gene, SLF1, by the yeast Pumilio-family protein Puf3p. PLoS One 6: e20441.

42. Wolfgang WJ, Miller TW, Webster JM, Huston JS, Thompson LM, Marsh JL, Messer A (2005). Suppression of Huntington's disease pathology in Drosophila by human single-chain Fv antibodies. Proc. Natl. Acad. Sci. USA 102: 11563-11568.

43. Weydt P, Pineda VV, Torrence AE, Libby RT, Satterfield TF, Lazarowski ER, Gilbert ML, Morton GJ, Bammler TK, Strand AD, Cui L, Beyer RP, Easley CN, Smith AC, Krainc D, Luquet S, Sweet IR, Schwartz MW, La Spada AR (2006). Thermoregulatory and metabolic defects in Huntington's disease transgenic mice implicate PGC-1alpha in Huntington's disease neurodegeneration. Cell Metab. 4: 349-362.

44. Weydt P, Soyal SM, Gellera C, Didonato S, Weidinger C, Oberkofler $\mathrm{H}$, Landwehrmeyer GB, Patsch W (2009). The gene coding for PGC1alpha modifies age at onset in Huntington's Disease. Mol. Neurodegener. 4: 3.

45. Taherzadeh-Fard E, Saft C, Andrich J, Wieczorek S, Arning L (2009). PGC-1alpha as modifier of onset age in Huntington disease. Mol. Neurodegener. 4: 10.

46. Weydt P, Soyal SM, Landwehrmeyer GB, Patsch W (2014). A single nucleotide polymorphism in the coding region of PGC-1alpha is a male-specific modifier of Huntington disease age-at-onset in a large European cohort. BMC Neurol. 14: 1.

47. Myers AM, Pape LK, Tzagoloff A (1985). Mitochondrial protein synthesis is required for maintenance of intact mitochondrial genomes in Saccharomyces cerevisiae. EMBO J. 4: 2087-2092. 
48. Kyryakov P, Beach A, Richard VR, Burstein MT, Leonov A, Levy S, Titorenko VI (2012). Caloric restriction extends yeast chronological lifespan by altering a pattern of age-related changes in trehalose concentration. Front. Physiol. 3: 256.

49. Guaragnella N, Zdralevic M, Lattanzio P, Marzulli D, Pracheil T, Liu Z, Passarella S, Marra E, Giannattasio S (2013). Yeast growth in raffinose results in resistance to acetic-acid induced programmed cell death mostly due to the activation of the mitochondrial retrograde pathway. Biochim. Biophys. Acta. 1833: 2765-2774.

50. Ocampo A, Barrientos A (2011). Quick and reliable assessment of chronological life span in yeast cell populations by flow cytometry. Mech. Ageing Dev. 132: 315-323.
51. Barrientos A (2002). In vivo and in organello assessment of OXPHOS activities. Methods 26: 307-316.

52. Tiefenbock SK, Baltzer C, Egli NA, Frei C (2010). The Drosophila PGC-1 homologue Spargel coordinates mitochondrial activity to insulin signalling. ЕMBO J. 29: 171-183

53. Ali YO, Escala W, Ruan K, Zhai RG (2011). Assaying locomotor, learning, and memory deficits in Drosophila models of neurodegeneration. J. Vis. Exp. 49: 2504.

54. Ruan K, Zhu Y, Li C, Brazill JM, Zhai RG (2015). Alternative splicing of Drosophila Nmnat functions as a switch to enhance neuroprotection under stress. Nat. Commun. 6: 10057. 